\title{
Whitman Family Genealogy
}

Paternal Grandparents

Jesse W. Whitman (29 January 1749-12 February 1803)

Hannah Brush (6 October 1753-6 January 1834)

Maternal Grandparents

Cornelius Van Velsor (1768-August 1837)

Naomi "Amy" Williams (1763-15 February 1826)

Parents

Walter Whitman Sr. (14 July 1789-11 July 1855)

m. 8 June 1816

Louisa Van Velsor (22 September 1795-23 May 1873)

Children

Jesse (2 March 1818-21 March 1870)

Walter (31 May 1819-26 March 1892) "Walt"

Mary Elizabeth (3 February 1821-6 August 1899)

m. 2 January 1840, Ansel Van Nostrand

George Van Nostrand (1841-?)

Fanny Van Nostrand (1843-?)

Louisa Van Nostrand (1845-?)

Ansel Van Nostrand (1847-?)

Minnie Van Nostrand (1857-?)

Hannah Louisa (28 November 1823-18 July 1908)

m. 16 March 1852, Charles Louis Heyde (1820-1892)

Infant (2 March 1825-14 September 1825)

Andrew Jackson (7 April 1827-3 December 1863) "Bunkum"

m. 1852? Nancy McClure (1834-?)

James Whitman (d. 1892) "Jimmy"

George Whitman (d. 1872) "Georgy"

Andrew Whitman (d. 1868) "Little Andrew" 
George Washington (28 November 1829-20 December 1901)

m. 14 April 1871, Louisa Orr Haslam (2 March 1842-9 August 1892) "Lou"

Walter Orr Whitman (4 November 1875-12 July 1876)

Thomas Jefferson (18 July 1833-25 November 1890) "Jeff"

m. 23 February 1859, Martha Mitchell (12 September 1836-19 February 1873) "Mattie"

Mannahatta (9 June 1860-3 September 1886) "Hattie"

Jessie Louisa (17 June 1863-1957) "California" or "Sis"

Edward (9 August 1835-30 November 1892) "Eddy" 


\section{Hannah Whitman Heyde

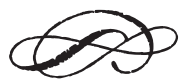


